\title{
ON THE PRIMARY COVERINGS OF FINITE SOLVABLE AND SYMMETRIC GROUPS
}

\author{
FRANCESCO FUMAGALLI AND MARTINO GARONZI
}

\begin{abstract}
A primary covering of a finite group $G$ is a family of proper subgroups of $G$ whose union contains the set of elements of $G$ having order a prime power. We denote with $\sigma_{0}(G)$ the smallest size of a primary covering of $G$, and call it the primary covering number of $G$. We study this number and compare it with its analogous $\sigma(G)$, the covering number, for the classes of groups $G$ that are solvable and symmetric.
\end{abstract}

\section{INTRODUCTION}

A covering of a finite group $G$ is a family of proper subgroups of $G$ whose union equals $G$. The covering number of $G$ is defined as the minimal size of a covering of $G$, and it is denoted by $\sigma(G)$ (by Cohn [1). A group admits a covering unless it is cyclic, in which case it is generally set $\sigma(G)$ to be equal to $\infty$ (with the convention that $n<\infty$ for every integer $n$ ). The covering number has been studied by many authors and in particular $\sigma(G)$ was determined when $G$ is solvable by Tomkinson [17. Theorem 2.2] and when $G$ is symmetric by Maróti [12, Theorem] (see also [16, [11] and [14]).

Given a subset $\Pi$ of $G$ we may be interested in the minimal number of proper subgroups of $G$ whose union contains $\Pi$. In this paper we focus on the set of primary elements, complementing the work done in 4. A primary element of $G$ is an element of $G$ whose order is some prime power. We define $G_{0}$ to be the set of primary elements of $G$ and a primary covering of $G$ to be a family of proper subgroups of $G$ whose union contains $G_{0}$. We set $\sigma_{0}(G)$ the smallest size of a primary covering of $G$. and call it the primary covering number of $G$. Observe that $G$ admits primary coverings if and only if $G$ is not a cyclic $p$-group for no prime $p$, so in this case we define $\sigma_{0}(G)=\infty$, with the convention that $n<\infty$ for every integer $n$. Clearly, we always have $\sigma_{0}(G) \leq \sigma(G)$. Moreover, a deep result (3, Theorem 1]) shows that a primary covering of any finite group is never a unique conjugacy class of a proper subgroup.

In this paper we study $\sigma_{0}(G)$ when $G$ is solvable and when $G$ is a symmetric group $S_{n}$.

Our main result about solvable groups is the following.

Date: April 5, 2021.

Francesco Fumagalli. Dipartimento di Matematica e Informatica "Ulisse Dini", viale Morgagni 67/A, 50134 Firenze, Italy. francesco.fumagalli@unifi.it. Member of INDAM.

Martino Garonzi. Universidade de Brasília, Campus Universitário Darcy Ribeiro, Departamento de Matemàtica Brasília - DF 70910-900, Brasil. mgaronzi@gmail.com. Supported by Fundação de Apoio à Pesquisa do Distrito Federal (FAPDF) - demanda espontânea 03/2016, and by Conselho Nacional de Desenvolvimento Científico e Tecnológico (CNPq) - Grant numbers 302134/2018-2, 422202/2018-5. 
Theorem 1. Let $G$ be a finite solvable group which is not a cyclic p-group, for every prime $p$. If $G / G^{\prime}$ is not a p-group, then $\sigma_{0}(G)=2$. Otherwise, $\sigma_{0}(G)=\sigma(G)$.

Our results on the primary covering number for $S_{n}$ can be summarized in the following statement.

Theorem 2. The following hold for $n \geq 3$.

(1) $\sigma_{0}\left(S_{3}\right)=4$ and $\sigma_{0}\left(S_{6}\right)=7$ (see Lemmas 8 and 11).

(2) If $n=2^{a}$ for some $a>1$, then $\sigma_{0}\left(S_{n}\right)=1+\frac{1}{2}\left(\begin{array}{c}n \\ n / 2\end{array}\right)$ (see Proposition 1 ).

(3) If $n \neq 3^{\epsilon} 2^{a}$, for $\epsilon \in\{0,1\}$ and $a>1$, then $\sigma_{0}\left(S_{n}\right)=1+\left(\begin{array}{c}n \\ n_{2}\end{array}\right)$, where $n_{2}$ denotes the maximum power of 2 that divides $n$ (see Proposition 4 and Lemma [10).

(4) If $n=3 \cdot 2^{a}$, with $a \geq 2$, then $c_{1} \leq \sigma_{0}\left(S_{n}\right) \leq c_{2}$, where

$$
\begin{aligned}
& c_{1}= \begin{cases}117 & \text { if } a=2, \\
1+\left(\begin{array}{c}
n-1 \\
2^{a}-1
\end{array}\right) & \text { if } a \geq 3,\end{cases} \\
& c_{2}=2+\left(\begin{array}{c}
n-1 \\
2^{a}-1
\end{array}\right)+\sum_{i=2}^{2^{a+1}}\left(\begin{array}{c}
n-i \\
2^{a-1}-1
\end{array}\right)
\end{aligned}
$$

(see Proposition 5 ).

As a comparison with the work done in [4, observe the following. Denoting by $\gamma_{0}(G)$ the normal primary covering number of a finite group $G$, that is the smallest natural number such that

$$
G_{0} \subseteq \bigcup_{i=1}^{\gamma_{0}(G)} \bigcup_{g \in G} H_{i}^{g}
$$

for some proper - pairwise non-conjugate - subgroups $H_{i}$ of $G$, by Propositions 1 and 5 in [4] we have that if $G$ is a group whose order is not a prime power and $G$ is either solvable or symmetric then $\gamma_{0}(G)=2$.

\section{Solvable groups}

In this section we determine the primary covering number for every finite solvable group $G$. For the basic results, as well as the notation, concerning solvable groups we refer the reader to [15, Chapter 5].

We start with the following trivial observations that hold in a general context.

Lemma 1. Assume that $G$ is a finite group.

(1) If $G / G^{\prime}$ is not a p-group, for some prime $p$, then $\sigma_{0}(G)=2$.

(2) If $N \unlhd G$, then $\sigma_{0}(G) \leq \sigma_{0}(G / N)$. Moreover, if $N$ is contained in the Frattini subgroup of $G$, then $\sigma_{0}(G)=\sigma_{0}(G / N)$.

(3) If $M$ is a maximal subgroup of $G$ such that $\sigma_{0}(M)>\sigma_{0}(G)$, then $M$ belongs to every minimal primary covering of $G$.

Proof. (1) We trivially have that $G_{0} \subseteq H \cup K$, where $H$ and $K$ are maximal subgroups containing $G^{\prime}$ of coprime indices.

(2) Since any primary covering of $G / N$ lifts to a primary covering of $G$, we have $\sigma_{0}(G) \leq \sigma_{0}(G / N)$. Also, any subgroup in a primary covering of $G$ can be replaced by a maximal subgroup containing it. Thus when $N \leq \Phi(G)$ we have $\sigma_{0}(G)=\sigma_{0}(G / N)$. 
(3) Let $M$ be a maximal subgroup of $G$ such that $\sigma_{0}(G)<\sigma_{0}(M)$ and let $H_{1}, \ldots, H_{n}$ be any primary covering of $G$ of size $n=\sigma_{0}(G)$. Of course, the family $\left\{H_{i} \cap M\right\}_{i=1}^{n}$ covers $M_{0}$ (the set of primary elements of $M$ ), so since $\sigma_{0}(M)>n$ we deduce that there exists $i \in\{1, \ldots, n\}$ such that $H_{i} \cap M=M$, in other words $M \leq H_{i}$. Being $M$ a maximal subgroup of $G$ we deduce $M=H_{i}$.

Lemma 2. Let $N$ be a complemented minimal normal subgroup of a solvable group $G$ and let $b$ be the number of complements of $N$ in $G$. If $b>1$ then $b \geq|N|$.

Proof. Let $E=\operatorname{End}_{G}(N)$. By a result of Gaschütz, [5, Satz 3], we know that $b=|N|^{\varepsilon}|E|^{\beta-1}$ where $\varepsilon$ is 0 or 1 according to whether $N$ is central or not in $G$, and $\beta$ is the number of non-Frattini chief factors $G$-isomorphic to $N$, in a chief series of $G$ starting with $N$. It follows that $b \geq|N|$ if $N$ is not central, and if $N$ is central then $|N|=p$ is a prime number and $E$ is the field with $p$ elements, so that $\beta \neq 1$ (being $b>1$ by hypothesis) hence $b \geq|E|=p=|N|$.

Recall Tomkinson's result ([17, Theorem 2.2]) which states that if $G$ is a finite solvable group then $\sigma(G)=q+1$ where $q$ is the order of the smallest chief factor of $G$ with more than one complement.

Proof of Theorem 1. Let $G$ be a non-cyclic finite solvable group. If $\left|G / G^{\prime}\right|$ is not a prime power, then by Lemma 1 (1) we have $\sigma_{0}(G)=2$. Thus assume that $G / G^{\prime}$ is a $p$-group for some prime $p$ and define $\alpha$ to be the smallest order of a chief factor of $G$ admitting more than one complement (this is well-defined because $G$ is not a cyclic $p$-group). We need to show that $\sigma_{0}(G)=1+\alpha$. By the aforementioned result of Tomkinson, $\sigma_{0}(G) \leq \sigma(G)=1+\alpha$. Let $N$ be a normal subgroup of $G$ such that $\sigma_{0}(G)=\sigma_{0}(G / N)$ with $|G / N|$ minimal with this property. Let $K / N$ be a minimal normal subgroup of $G / N$, then $\sigma_{0}(G / N)<\sigma_{0}(G / K)$, hence by Lemma 1 (2) $K / N$ admits a complement $M / N$ in $G / N$ which is a maximal subgroup. Being $\sigma_{0}(G / N)<\sigma_{0}(G / K)=\sigma_{0}(M / N)$, we deduce that $M / N$ appears in every minimal primary covering of $G / N$, hence all $b$ complements of $K / N$ in $G / N$ appear in a fixed minimal primary covering of $G / N$. However, no element of $K / N$ belongs to any complement of $K / N$, hence $\sigma_{0}(G / N) \geq 1+b$. If $b \neq 1$, then $b \geq|K / N|$ by Lemma 2 and this implies the result. Assume now $b=1$. Then $G / N$ is a direct product $K / N \times M / N$ hence $K / N$, being a central chief factor, is cyclic of prime order and, being an epimorphic image of the $p$-group $G / G^{\prime}$, we deduce that $|K / N|=p$. Moreover $M / N$ is nontrivial, because $G / N \neq C_{p}$ (being $\left.\sigma_{0}(G)=\sigma_{0}(G / N)\right)$, so $M / N$ has nontrivial abelianization. Since $G / G^{\prime}$ is a $p$-group it follows that $M / N$ projects onto $C_{p}$. This $C_{p}$ is therefore a chief factor above $K / N$ which is $G$-isomorphic to $K / N$. In particular, the number of non-Frattini chief factors $G$-isomorphic to $C_{p}$ is $\beta \geq 2$. This contradicts the formula in the proof of Lemma 2 .

\section{Symmetric GROUPS}

We introduce some notation that will be frequently used. Let $n$ be a positive integer and set $\Omega:=\{1,2, \ldots, n\}$ on which the symmetric group $S_{n}$ acts naturally. Given natural numbers $k \geq 1$ and $x_{1} \geq x_{2} \geq \ldots \geq x_{k} \geq 1$ such that $\sum_{i=1}^{k} x_{i}=n$, we denote with $\left(x_{1}, x_{2}, \ldots, x_{k}\right)$ both the partition of $n$ whose parts are precisely the $x_{i}$ and the set of all permutations in $S_{n}$ having as cyclic type this partition. In particular, $(n)$ denotes the set of $n$-cycles of $S_{n}$. 
The maximal subgroups of $S_{n}$ split in three different classes, according to their action on $\Omega$ : intransitive, imprimitive and primitive subgroups (see [2]).

Any intransitive maximal subgroup is the setwise stabilizer of a set of cardinality $m$, for some $1 \leq m<n / 2$. In particular, any such subgroup is conjugate to the stabilizer of the set $\{1,2, \ldots, m\} \subseteq \Omega$, which we denote with $X_{m}$. It is therefore isomorphic to $S_{m} \times S_{n-m}$ and its index is $\left(\begin{array}{c}n \\ m\end{array}\right)$. We set

$$
\mathcal{X}_{m}=\left\{\text { conjugates of } X_{m} \simeq S_{m} \times S_{n-m}\right\}
$$

and

$$
\mathcal{X}=\bigcup_{1 \leq m<n / 2} \mathcal{X}_{m}=\left\{\text { intransitive maximal subgroups of } S_{n}\right\} .
$$

The imprimitive maximal subgroups of $S_{n}$ are the stabilizers of partitions of $\Omega$ into equal-sized subsets. If $d$ is any proper nontrivial divisor of $n$ we set $W_{d}$ the stabilizer of the partition

$$
\{\{1,2, \ldots, d\},\{d+1, d+2, \ldots, 2 d\}, \ldots,\{n-d+1, n-d+2, \ldots, n\}\} .
$$

Note that $W_{d}$ is isomorphic to the wreath product $S_{d}<S_{n / d}$, and it has index $\frac{n !}{(d !)^{n / d} \cdot(n / d) !}$. Also note that any imprimitive maximal subgroup of $S_{n}$ is conjugate to $W_{d}$, for some proper nontrivial divisor $d$ of $n$. We set

$$
\mathcal{W}_{d}=\left\{\text { conjugates of } W_{d} \simeq S_{d} 2 S_{n / d}\right\}
$$

and

$$
\mathcal{W}=\bigcup_{1<d \mid n, d \neq n} \mathcal{W}_{d}=\left\{\text { imprimitive maximal subgroups of } S_{n}\right\} .
$$

Finally set

$$
\mathcal{P}=\left\{\text { proper primitive maximal subgroups of } S_{n}\right\}
$$

where proper means that both $S_{n}$ and $A_{n}$ are not members of $\mathcal{P}$.

Lemma 3. If $a>b$ are positive integers then

$$
a !^{b} b ! \geq b !^{a} a !
$$

with equality if and only if $b=1$.

Proof. If $b=1$ we have equality, now assume $b \geq 2$, so that $a \geq 3$. Since the stated inequality is equivalent to

$$
\frac{\ln (a !)}{a-1} \geq \frac{\ln (b !)}{b-1}
$$

it is enough to prove that the function $\ln (a !) /(a-1)$ is increasing, hence we may assume $b=a-1$. So we need to prove that $a !^{a-2} \geq(a-1) !^{a-1}$ which is equivalent to $a^{a-1} \geq a$ !, which is actually a strict inequality being $a \geq 3$.

The following lemmas are part of [13, Corollary 1.2 and Lemma 2.1].

Lemma 4 (Maróti Lemma 2.1, On the orders of primitive groups). Let $m>1$ be an integer and suppose $m=a_{1} b_{1}=a_{2} b_{2}$ with $a_{1}, a_{2}, b_{1}, b_{2}$ positive integers at least $2, b_{1} \geq a_{1}, b_{2} \geq a_{2}, a_{1} \leq a_{2}$ and (consequently) $b_{1} \geq b_{2}$. Then

$$
b_{1} !^{a_{1}} a_{1} ! \geq b_{2} !^{a_{2}} a_{2} \text { ! }
$$

with equality if and only if $a_{1}=a_{2}$ and $b_{1}=b_{2}$. Moreover if $p$ is the smallest prime divisor of $m$ and $d$ is any divisor of $m$ with $1<d<m$ then

$$
(m / p) !^{p} p ! \geq(m / d) !^{d} d !
$$


with equality if and only if $d=p$.

Proof. Observe that since $b_{2} \geq a_{2} \geq 2$ we have $b_{2}^{b_{2}} \geq b_{2} ! b_{2} \geq b_{2} ! a_{2}$.

$$
\begin{aligned}
b_{1} !^{a_{1}} a_{1} ! & \geq b_{2} !^{a_{1}}\left(b_{2}+1\right)^{a_{1}} \cdots b_{1}^{a_{1}} a_{1} ! \\
& \geq b_{2} !^{a_{1}} a_{1} ! b_{2}^{a_{1}\left(b_{1}-b_{2}\right)} \\
& \geq b_{2} !^{a_{1}} a_{1} !\left(b_{2} ! a_{2}\right)^{\left(a_{1} / b_{2}\right)\left(b_{1}-b_{2}\right)} \\
& =b_{2} !^{a_{1}} a_{1} !\left(b_{2} ! a_{2}\right)^{\left(a_{2}-a_{1}\right)} \\
& \geq b_{2} !^{a_{1}} a_{1} ! b_{2} !^{a_{2}-a_{1}}\left(a_{1}+1\right) \cdots a_{2} \\
& =b_{2} !^{a_{2}} a_{2} !
\end{aligned}
$$

If equality holds then all of the above inequalities are equalities and it is easy to deduce that $a_{1}=a_{2}$ and consequently $b_{1}=b_{2}$. To deduce the last statement, observe that it is trivial if $m=p$ so assume this is not the case, so that $p^{2} \leq m$. Choose $a_{1}=p, b_{1}=m / p$, then $a_{1} \leq b_{1}$ and the inequality $a_{1} \leq a_{2}$ will be true for every choice of a divisor $a_{2}>1$ of $m$, by minimality of $p$. If $d^{2} \leq m$ then choose $a_{2}=d$, proving the strict inequality with equality if and only if $d=p$. If $d^{2}>m$ then $d>m / d$ hence Lemma 3 implies that $d !^{m / d}(m / d) ! \geq(m / d) !^{d} d$ !, so it is enough to show that $(m / p) !^{p} p ! \geq d !^{m / d}(m / d) !$, which follows from the above choosing $a_{2}=m / d$, and again by Lemma 3 equality does not hold in this case being $m / d \neq 1$.

The orders of the different primitive and imprimitive maximal subgroups of $S_{n}$ can be compared as in the following lemma.

Lemma 5. Let $n \geq 2$, then the following hold.

(1) For every proper nontrivial divisor $d$ of $n$, we have

$$
\left|W_{n / p}\right|=(n / p) !^{p} p ! \geq(n / d) !^{d} d !=\left|W_{d}\right|,
$$

where $p$ is the smallest prime divisor of $n$, and equality holds if and only if $d=p$

(2) if $P \in \mathcal{P}$, then $|P|<3^{n}$, and when $n>24$, then $|P|<2^{n}$.

In particular, for $n \geq 12$ every $M \in \mathcal{W} \cup \mathcal{P}$ has order

$$
|M| \leq 2(\lfloor n / 2\rfloor) !(n-\lfloor n / 2\rfloor) !
$$

with equality if and only if $M \in \mathcal{W}_{n / 2}$.

Proof. (1) follows from Lemma 4, and (2) is [13, Corollary 1.2].

We now prove the last part of the Lemma.

Let first $M \in \mathcal{P}$. By (2) the order of $M$ is bounded above by $3^{n}$. Recall Stirling's bound $k !>e(k / e)^{k}$, which holds for every $k \geq 2$ and can be proved by noting that

$$
\frac{k^{k}}{k !}=\frac{k^{k-1}}{(k-1) !}=\prod_{i=1}^{k-1}\left(\frac{i+1}{i}\right)^{i}<\prod_{i=1}^{k-1}\left(e^{1 / i}\right)^{i}=e^{k-1} .
$$

We deduce that

$$
2(\lfloor n / 2\rfloor) !(n-\lfloor n / 2\rfloor) !>g(n)= \begin{cases}2 e^{2}\left(\frac{n}{2 e}\right)^{n} & \text { if } n \text { is even, } \\ e(n+1)\left(\frac{n-1}{2 e}\right)^{n-1} & \text { if } n \text { is odd. }\end{cases}
$$


Computation shows that for every $n \geq 14$ the function $g(n) \geq 3^{n}$. The cases $n=12$ and $n=13$ can be done by inspection and the proof for the primitive case is completed.

Assume now that $M \in \mathcal{W}$, in particular $m$ is not a prime number. Then by (1) we have $|M| \leq\left|W_{n / p}\right|$, where $p$ is the smallest prime number that divides $n$. The result is therefore trivial if $n$ is even. Let $n$ be odd. We need to prove that

$$
R=\frac{(n+1)(((n-1) / 2) !)^{2}}{(n / p) !^{p} p !}>1 .
$$

Observe that, being $p^{2} \leq n$, the map $x \mapsto x^{n-1} / n^{x}$ is an increasing function in the interval $1 \leq x \leq p$, so that $p^{n-1} / n^{p} \geq 3^{n-1} / n^{3}$. Using the inequalities $e(n / e)^{n} \leq n ! \leq e n(n / e)^{n}$ we see that

$$
\begin{aligned}
R & \geq \frac{e^{2}(n+1)\left(\frac{n-1}{2 e}\right)^{n-1}}{\operatorname{epn}^{p}\left(\frac{n}{e p}\right)^{n}}=2 e^{2} \cdot \frac{n+1}{n-1} \cdot \frac{p^{n-1}}{n^{p}} \cdot\left(\frac{n-1}{2 n}\right)^{n} \\
& >2 e^{2} \cdot \frac{3^{n-1}}{n^{3}} \cdot\left(\frac{n-1}{2 n}\right)^{n}=\frac{2 e^{2}}{3 n^{3}}\left(\frac{3(n-1)}{2 n}\right)^{n} \geq 1
\end{aligned}
$$

whenever $n \geq 22$. The case $12 \leq n \leq 21$ can be done by inspection.

In the sequel we will need the following result.

Lemma 6. Let $n$ be even and $W \in \mathcal{W}_{n / 2}$. Assume that $n=\sum_{i=1}^{k} 2^{a_{i}}$ is a partition of $n$ with $a_{1} \geq a_{2} \geq \ldots \geq a_{k} \geq 1$ that does not contain subpartitions of $n / 2$, and let $\Pi$ be the conjugacy class of elements of $S_{n}$ of type $\left(2^{a_{1}}, 2^{a_{2}}, \ldots, 2^{a_{k}}\right)$. Then

$$
|W \cap \Pi|=\frac{|\Pi|}{\left|S_{n}: W\right|} \cdot 2^{k-1} .
$$

In particular, when $n$ is a power of 2 and $\Pi=(n)$, the set of $n$-cycles, then

$$
|W \cap \Pi|=\frac{|W|}{n}=(n / 2) !(n / 2-1) !
$$

Proof. Double counting the size of the set

$$
\left\{(x, W) \mid x \in \Pi \cap W, W \in \mathcal{W}_{n / 2}\right\},
$$

we find that

$$
|W \cap \Pi|=\frac{|\Pi|}{|G: W|} \cdot r,
$$

where $r$ is the number of elements of $\mathcal{W}_{n / 2}$ containing a fixed element of $\Pi$. By the assumption that the partition defining $\Pi$ does not contain partitions of $n / 2$ as subpartitions, the elements of $\Pi \cap W$ move the two imprimitivity blocks of $W$, hence $r=2^{k-1}$, since no cycle can fix either block, and, once we split up the elements in the $2^{a_{1}}$-cycle, we have two choices for each $2^{a_{j}}$-cycle, $2 \leq j \leq k$.

Moreover, we will make use of the following notation and terminology introduced in 12 .

Definition 1. Let $\Pi$ be a set of permutations of $S_{n}$. We will say that a collection $\mathcal{H}=\left\{H_{1}, \ldots, H_{m}\right\}$ of $m$ proper subgroups of $S_{n}$ is definitely unbeatable on $\Pi$ if the following three conditions hold:

(1) $\Pi \subseteq \bigcup_{i=1}^{m} H_{i}$, 
(2) $\Pi \cap H_{i} \cap H_{j}=\varnothing$ for every $i \neq j$,

(3) $|M \cap \Pi| \leq\left|H_{i} \cap \Pi\right|$ for every $1 \leq i \leq m$ and every proper subgroup $M$ of $S_{n}$ not belonging to $\mathcal{H}$.

If $\mathcal{H}$ is definitely unbeatable on $\Pi$, then $|\mathcal{H}|=\sigma(\Pi)$, where $\sigma(\Pi)$ denotes the least integer $m$ such that $\Pi$ is a subset of the union of $m$ proper subgroups of $S_{n}$. Moreover, we also say that $\mathcal{H}$ is strongly definitely unbeatable on $\Pi$ if the three conditions above hold and the third one always holds with strict inequalites. Note that in the case when $\mathcal{H}$ consists of maximal subgroups and it is strongly definitely unbeatable on $\Pi$, then $\mathcal{H}$ is the unique minimal cover of the elements of $\Pi$ that uses only maximal subgroups (see also [16, Lemma 3.1]).

We start our considerations on the primary covering number of $S_{n}$ by producing a general upper bound. Here and in the following if $p$ is any prime, we define $n_{p}$ to be the $p$-part of $n$, that is the maximum power of $p$ that divides $n$.

\section{Lemma 7 .}

(1) If $n$ is a power of 2 , then $\sigma_{0}\left(S_{n}\right) \leq 1+\frac{1}{2}\left(\begin{array}{c}n \\ n / 2\end{array}\right)$,

(2) if $n$ is not a power of 2 , then $\sigma_{0}\left(S_{n}\right) \leq 1+\left(\begin{array}{c}n \\ n_{2}\end{array}\right)$.

Proof. Note that in any case the alternating groups $A_{n}$ contains every permutation of odd order, therefore in order to exhibit a primary covering for $S_{n}$, we may add to $\left\{A_{n}\right\}$ those subgroups that contain 2-elements (that are odd permutations).

(1) Assume $n=2^{a}$ with $a \geq 2$. Then every 2-element of $S_{n}$ stabilizes a 2-block partition of $\Omega$ and therefore every 2-element belongs to an imprimitive maximal subgroup of type $\mathcal{W}_{n / 2}$. Since the number of such partitions (and subgroups) is $\frac{1}{2}\left(\begin{array}{c}n \\ n / 2\end{array}\right)$ the lemma is proved in this case.

(2) The number of subsets of order $n_{2}$ of the set $\Omega=\{1,2, \ldots, n\}$ is $\left(\begin{array}{c}n \\ n_{2}\end{array}\right)$ which is an odd number (see for instance [9, Lemma 1.8]), and it is easy to prove by induction that every 2-element of $S_{n}$ belongs to some stabilizer $S_{\Delta}$ with $\Delta$ a subset of $\Omega$ of cardinality $n_{2}$, in other words if we write $n$ as a sum of distinct powers of 2 then there exists a subsum that equals $n_{2}$. Since the number of these stabilizers is exactly $\left(\begin{array}{c}n \\ n_{2}\end{array}\right)$ also this point is proved.

We already have enough ingredients to complete the proof in the case $n=2^{a}$, with $a \geq 2$.

Proposition 1. If $n=2^{a} \geq 4$ then $\sigma_{0}\left(S_{n}\right)=1+\frac{1}{2}\left(\begin{array}{c}n \\ n / 2\end{array}\right)$ and a minimal primary covering is given by $\left\{A_{n}\right\} \cup \mathcal{W}_{n / 2}$.

Proof. A direct inspection shows that $\sigma_{0}\left(S_{4}\right)=4=1+\frac{1}{2}\left(\begin{array}{l}4 \\ 2\end{array}\right)$. Thus in the following we assume $a>2$.

By Lemma 7 we know that $\sigma_{0}\left(S_{n}\right) \leq 1+\frac{1}{2}\left(\begin{array}{c}n \\ n / 2\end{array}\right)$.

Assume that $H$ is a maximal subgroup of $S_{n}$. Then either $H \cap(n)=\varnothing$, or $H$ is an imprimitive subgroup, or by [10, Theorem 3] the subgroup $H$ satisfies $\mathrm{PGL}_{d}(q) \leq H \leq \mathrm{P \Gamma L}_{d}(q)$, where $n=\left(q^{d}-1\right) /(q-1)$ for some $d \geq 2$. Note that in this last case we necessarily have $d=2$ and $q=2^{a}-1$ a Mersenne prime. To see this observe that from the equality $2^{a}=\left(q^{d}-1\right) /(q-1)$ one easily deduces that $d=2$ so that $2^{a}=q+1$, now $q$ cannot be a square since $2^{a}-1 \equiv 3$ $\bmod 4$, and if $q=p^{m}$ is an odd power of the prime $p$, the usual factorization 
of $x^{m}+1=(x+1)\left(x^{m-1}-x^{m-2}+\ldots+1\right)$ implies that $q$ must be a prime. By Lemma 6] and the fact the elements of order $n$ in $\operatorname{PGL}(2, q)$ are in number of $2^{a-2}\left(2^{a}-1\right)\left(2^{a}-2\right)$ (use [8, II, Satz 7.3$\left.]\right)$, we deduce in any case that

$$
|H \cap(n)| \leq(n / 2) !(n / 2-1) \text { ! }
$$

with equality if and only if $H \in \mathcal{W}_{n / 2}$. This shows that the set $\mathcal{W}_{n / 2}$ is strongly definitely unbeatable on $\Pi=(n)$, and therefore we obtain that

$$
\sigma_{0}\left(S_{n}\right) \geq \frac{1}{2}\left(\begin{array}{c}
n \\
n / 2
\end{array}\right)
$$

To complete this case, assume that $\sigma_{0}\left(S_{n}\right)=\frac{1}{2}\left(\begin{array}{c}n \\ n / 2\end{array}\right)$. Then, by the above, the collection $\mathcal{W}_{n / 2}$ must be the unique minimal primary covering for $S_{n}$. By Bertrand's postulate there is a prime number $p$ between $n / 2$ and $n$; we reach a contradiction by noting that $p$-cycles do not belong to imprimitive subgroups of type $\mathcal{W}_{n / 2}$.

We assume now that $n \notin\left\{3^{\epsilon} \cdot 2^{a} \mid \epsilon \in\{0,1\}, a \geq 0\right\}$. We deal separately with the case $n=5$.

Lemma 8. For $n=5$ we have that $\sigma_{0}\left(S_{5}\right)=1+\left(\begin{array}{l}5 \\ 1\end{array}\right)=6$ and $\left\{A_{5}\right\} \cup \mathcal{X}_{1}$ is the unique minimal primary covering of $S_{5}$.

Proof. We already know that $\left\{A_{5}\right\} \cup \mathcal{X}_{1}$ is a primary covering for $S_{5}$ and therefore $\sigma_{0}\left(S_{5}\right) \leq 6$. Assume by contradiction that $\mathcal{C}$ is a primary covering of smaller cardinality. Inside $S_{5}$ there are six subgroups of order 5 , therefore, as $|\mathcal{C}| \leq 5$, there exists one element of $\mathcal{C}$ containing at least two different Sylow 5-subgroups. But the only proper subgroup of $S_{5}$ containing more than one subgroup of order 5 is $A_{5}$. Thus $A_{5} \in \mathcal{C}$ and the remaining members of $\mathcal{C}$ cover all of the odd 2-elements of $S_{5}$, which are thirty 4-cycles and ten 2-cycles. Any maximal subgroup isomorphic to $S_{4}$ contains precisely six 4-cycles and six 2-cycles, any $S_{3} \times S_{2}$ contains no 4cycles and four 2-cycles and any Frobenius group $5: 4$ contains ten 4 -cycles and no 2-cycles. Therefore, if we assume that $\mathcal{C}$ contains respectively $a_{1}$ subgroups in $\mathcal{X}_{1}$ (that is isomorphic to $S_{4}$ ), $a_{2}$ subgroups in $\mathcal{X}_{2}$ (that is isomorphic to $S_{3} \times S_{2}$ ), and $a_{3}$ primitive subgroups isomorphic to $5: 4$, we obtain the following system of Diophantine inequalities

$$
\left\{\begin{array}{l}
a_{1}+a_{2}+a_{3} \leq 4 \\
3 a_{1}+5 a_{3} \geq 15 \\
6 a_{1}+4 a_{2} \geq 10
\end{array}\right.
$$

The only integer solution of this system is $\left(a_{1}, a_{2}, a_{3}\right)=(2,0,2)$, but then if $\operatorname{Stab}_{S_{5}}(i)$ and $\operatorname{Stab}_{S_{5}}(j)$ are the two elements of $\mathcal{X}_{1}$ in $\mathcal{C}$ we have that the permutation $(i j)$ is not covered by elements of $\mathcal{C}$, which is a contradiction.

Let $n \geq 7$ and $n \notin\left\{3^{\epsilon} \cdot 2^{a} \mid \epsilon \in\{0,1\}, a \geq 0\right\}$ and write the 2-adic expansion of $n$ as

$$
n=2^{a_{1}}+2^{a_{2}}+\ldots+2^{a_{t}},
$$

where $a_{1}>a_{2}>\ldots>a_{t} \geq 0$ and $t \geq 2$. Note that $n_{2}=2^{a_{t}}$ and also that when $t=2$ then $a_{1} \geq a_{2}+2$.

We define $\Pi$ to be the following conjugacy class of permutations

$$
\Pi= \begin{cases}\left(2^{a_{1}}, 2^{a_{2}}, \ldots, 2^{a_{t}}\right) & \text { if } n \neq t(\bmod 2), \\ \left(2^{a_{1}-1}, 2^{a_{1}-1}, 2^{a_{2}}, \ldots, 2^{a_{t}}\right) & \text { if } n \equiv t(\bmod 2) .\end{cases}
$$


The set $\Pi$ consists of odd permutations, that is $A_{n} \cap \Pi=\varnothing$.

The computation of $\sigma_{0}\left(S_{n}\right)$ in this case depends on the following proposition.

Proposition 2. Assume that $n \notin\left\{3^{\epsilon} \cdot 2^{a} \mid \epsilon \in\{0,1\}, a \geq 1\right\}$. If $n$ is odd and $n \geq$ 15 or if $n$ is even, $n \geq 22$ and $n \neq 40$, the collection of subgroups $\mathcal{X}_{n_{2}}$ is strongly definitely unbeatable on $\Pi$.

For the proof of Proposition 2 we need the following number-theoretic result.

Lemma 9. Using the above assumptions and notation, define

$$
s= \begin{cases}\sum_{i=1}^{t} a_{i}+a_{1}-1 & \text { if } n \equiv t(\bmod 2) \\ \sum_{i=1}^{t} a_{i} & \text { if } n \neq \equiv t(\bmod 2)\end{cases}
$$

and

$$
f(n)=2^{s+1} \frac{\left(\begin{array}{c}
n \\
n_{2}
\end{array}\right)}{\left(\begin{array}{c}
n \\
\lfloor n / 2\rfloor
\end{array}\right)} .
$$

Then

$$
f(n)<1
$$

holds if and only if either $n$ is odd and $n \geq 15$ or $n$ is even, $n \geq 22$ and $n \neq 40$.

Proof. Since $a_{i} \leq a_{1}-(i-1)$ for every $i=1,2, \ldots, t$, we have

$$
s \leq \sum_{i=1}^{t} a_{i}+a_{1}-1 \leq t a_{1}+a_{t}-1-\frac{(t-2)(t-1)}{2} .
$$

The coefficient $a_{t}$ satisfies $a_{t} \leq a_{1}+1-t$ and therefore

$$
s \leq \begin{cases}t a_{1} & \text { if } n \text { is odd } \\ (t+1) a_{1}-2 & \text { if } n \text { is even }\end{cases}
$$

where we used the fact that $\left(t^{2}-3 t+2\right) / 2 \geq 0$ and $\left(t^{2}-t+2\right) / 2 \geq 2$ for every $t \geq 2$. Set $l=\log _{2}(n) \geq a_{1}$. Being $t \leq a_{1}-a_{t}+1$, and according to our assumptions $n \geq 15$ if $n$ is odd and $n \geq 22$ if $n$ is even, we have that $t \leq l$ if $n$ is odd and $t \leq l-1$ if $n$ is even. Thus we obtain

$$
s \leq \begin{cases}l^{2} & \text { if } n \text { is odd } \\ l^{2}-2 & \text { if } n \text { is even }\end{cases}
$$

By considering the binomial expansion of $(1+1)^{n}$ we have that $\left(\begin{array}{c}n \\ \lfloor n / 2\rfloor\end{array}\right) \geq \frac{2^{n}}{n+1}$, that is, being $l \leq \log _{2}(n+1) \leq l+1$,

$$
\left(\begin{array}{c}
n \\
\lfloor n / 2\rfloor
\end{array}\right) \geq 2^{n-(l+1)} .
$$

We distinguish now the different cases.

Case $n$ odd.

Then $\left(\begin{array}{c}n \\ n_{2}\end{array}\right)=n=2^{l}$ and (2) and (3) imply that

$$
f(n) \leq 2^{l^{2}+2 l+2-n} .
$$

We have that $l^{2}+2 l+2-n<0$ for every $n \geq 43$.

The cases $15 \leq n \leq 41$ can be checked by direct computation. (Note that $f(n)>1$ 
for $5 \leq n \leq 13)$.

Case $n$ even.

Assume first that $t \geq 4$.

Then $n \geq 2^{a_{t}}\left(1+2+\ldots+2^{t-1}\right)=n_{2}\left(2^{t}-1\right) \geq 15 n_{2}$, hence $n_{2} \leq\lfloor n / 15\rfloor$ and

$$
\left(\begin{array}{c}
n \\
n_{2}
\end{array}\right) \leq\left(\begin{array}{c}
n \\
\lfloor n / 15\rfloor
\end{array}\right) \text {. }
$$

Note that if $k$ is any non-negative integer then $k^{k} / k !<e^{k}$ by the well-known series expansion of the exponential function, therefore if $k \leq n$ then

$$
\left(\begin{array}{l}
n \\
k
\end{array}\right)=\frac{n}{k} \cdot \frac{n-1}{k} \cdot \ldots \cdot \frac{n-k+2}{k} \cdot \frac{n-k+1}{k} \cdot \frac{k^{k}}{k !}<(n e / k)^{k} .
$$

Using the fact that for every real number $x$ we have $x-1 \leq\lfloor x\rfloor \leq x$, we deduce that

$$
\left(\begin{array}{c}
n \\
n_{2}
\end{array}\right) \leq\left(\frac{15 e n}{n-15}\right)^{n / 15}=2^{\frac{n}{15} \log _{2}\left(\frac{15 e n}{n-15}\right)}
$$

Combining (21), (3) and (4) we have that

$$
f(n) \leq 2^{l^{2}+l-n\left(1-\frac{1}{15} \log _{2}\left(\frac{15 e n}{n-15}\right)\right) .} .
$$

Note that $f(n)<1$ if $l^{2}+l-n\left(1-\frac{1}{15} \log _{2}\left(\frac{15 e n}{n-15}\right)\right)<0$, which is true for every $n \geq 72$. The cases $22 \leq n \leq 70$, with $t \geq 4$, can be checked by a direct computation.

Let now $t=2$ or $t=3$.

In both cases we have that the value of $s$ is bounded from above by $3 l-3$, because when $t=2$ then $a_{1} \geq a_{2}+2$ and so

$$
s=2 a_{1}+a_{2}-1 \leq 3 a_{1}-3 \leq 3 l-3
$$

and when $t=3$ then

$$
s=a_{1}+a_{2}+a_{3} \leq a_{1}+a_{1}-1+a_{1}-2 \leq 3 l-3 .
$$

Moreover, when $t=2$, since $a_{1} \geq a_{2}+2$, we have that $n_{2} \leq\lfloor n / 5\rfloor$. Therefore, by considerations analogous to the ones above, we have that $f(n)<1$ if

$$
\begin{aligned}
& 4 l-1-n\left(1-\frac{1}{7} \log _{2}\left(\frac{7 e n}{n-7}\right)\right)<0 \text { when } t=3, \\
& 4 l-1-n\left(1-\frac{1}{5} \log _{2}\left(\frac{5 e n}{n-5}\right)\right)<0 \text { when } t=2 .
\end{aligned}
$$

Now (5) holds for every $n \geq 62$, while (6) holds for every $n \geq 114$.

As above, the intermediate cases can be checked by computation, the only exceptions being all $n$ even with $n \leq 20$ and $n=40$.

Proof of Proposition 圆, To prove that $\mathcal{X}_{n_{2}}$ is strongly definitely unbeatable on $\Pi$ we need to show that the three conditions of the Definition 1 are satisfied. Conditions (1) and (2) are straightforward (note that when $t=2$ we have that $a_{1}-1>a_{2}$ ). 
We show condition (3), that is, for every $X_{n_{2}} \in \mathcal{X}_{n_{2}}$ and every maximal subgroup $M$ of $S_{n}$, which is not the stabilizer of a $n_{2}$-subset, the proportion

$$
c(M):=\frac{|M \cap \Pi|}{\left|X_{n_{2}} \cap \Pi\right|}=\frac{|M|}{\left|X_{n_{2}}\right|} m_{M}<1,
$$

where $m_{M}$ denotes the number of conjugates of $M$ containing a fixed element of $\Pi$. The above expression for $c(M)$ was obtained via a double counting, as in the proof of Proposition 1, applied to compute both $|M \cap \Pi|$ and $\left|X_{n_{2}} \cap \Pi\right|$, keeping in mind that a given element of $\Pi$ belongs to exactly one member of $\mathcal{X}_{n_{2}}$.

Assume first that $M$ is an intransitive maximal subgroup not in $\mathcal{X}_{n_{2}}$.

If $M \cap \Pi \neq \varnothing$ we necessarily have that $M$ is the stabilizer of a union of disjoint 2 -power sized subsets, say $A_{i}$ for some $i \geq 1$. Since $|M \cap \Pi| \leq\left|\operatorname{Stab}\left(A_{1}\right) \cap \Pi\right|$, we can assume that $M$ coincides with the stabilizer of a single subset of cardinality some power of 2 , say $2^{c}$, with $n_{2}<2^{c}<n / 2$, being $2^{a_{1}} \geq n / 2$, and therefore $c \in\left\{a_{2}, \ldots, a_{t-1}\right\}$ when $n \not \equiv t(\bmod 2)$ and $c \in\left\{a_{1}-1, a_{2}, \ldots, a_{t-1}\right\}$ when $n \equiv t$ $(\bmod 2)$. Now, except in the case $n \equiv t(\bmod 2)$ and $c=a_{1}-1$, we have that $m_{M}=1$ and

$$
c(M)=\frac{\left(\begin{array}{c}
n \\
n_{2}
\end{array}\right)}{\left(\begin{array}{c}
n \\
2^{c}
\end{array}\right)}<1,
$$

since $n_{2}=2^{a_{t}}<2^{c}$. Otherwise $m_{M}=2$ and, since $a_{t} \leq a_{1}-2$, we obtain

$$
c(M)=2 \cdot \frac{\left(\begin{array}{c}
n \\
2^{a_{t}}
\end{array}\right)}{\left(\begin{array}{c}
n \\
2^{a_{1}-1}
\end{array}\right)} \leq 2 \cdot \frac{\left(\begin{array}{c}
n \\
2^{a_{1}-2}
\end{array}\right)}{\left(\begin{array}{c}
n \\
2^{a_{1}-1}
\end{array}\right)}<1 .
$$

Assume now that $M$ is a primitive or an imprimitive maximal subgroup of $S_{n}$. Then by Lemma 5 .

while

$$
|M \cap \Pi| \leq|M| \leq \frac{2 n !}{\left(\begin{array}{c}
n \\
\lfloor n / 2\rfloor
\end{array}\right)},
$$

$$
\left|X_{n_{2}} \cap \Pi\right|=\frac{n_{2} !\left(n-n_{2}\right) !}{2^{s}},
$$

where $s=\sum_{i=1}^{t} a_{i}$ if $n \not \equiv t(\bmod 2)$, and $s=\sum_{i=1}^{t} a_{i}+a_{1}-1$ if $n \equiv t(\bmod 2)$. Therefore

$$
c(M)=\frac{|M \cap \Pi|}{\left|X_{n_{2}} \cap \Pi\right|} \leq \frac{|M|}{\left|X_{n_{2}} \cap \Pi\right|} \leq 2^{s+1} \cdot \frac{\left(\begin{array}{c}
n \\
n_{2}
\end{array}\right)}{\left(\begin{array}{c}
n \\
\lfloor n / 2\rfloor
\end{array}\right)}
$$

Lemma 9 proves that $c(M)<1$, whenever $n$ is odd and $n \geq 15$, or $n$ is even and $n \geq 22$ and $n \neq 40$.

We treat now the case when $n \leq 20$ or $n=40$.

Proposition 3. For $n \in\{5,7,9,10,11,13,14,18,20,40\}$, the collection of subgroups $\mathcal{X}_{n_{2}}$ is strongly definitely unbeatable on $\Pi$ if and only if $n \notin\{5,10\}$.

Proof. As in the proof of Proposition 2, the conditions (1) and (2) of Definition 1 are straightforward to prove, so we limit ourselves to show that condition (3) holds.

In the sequel, denote by $M$ a maximal subgroup of $S_{n}$ not in $\mathcal{X}_{n_{2}} \cup\left\{A_{n}\right\}$.

If $M$ is intransitive the inequality $|M \cap \Pi| \leq\left|X_{n_{2}} \cap \Pi\right|$ holds in all cases, with equality if and only if $M \in \mathcal{X}_{n_{2}}$ (see the proof of Proposition 21). So we may concentrate on the imprimitive and primitive maximal subgroups. We treat the various cases separately. 
Case $n=5$. Then $\Pi=(4,1)$ and if we take $M$ to be a primitive subgroup isomorphic to the Frobenius group $5: 4$, we have that

$$
|M \cap \Pi|=10>6=\left|X_{n_{2}} \cap \Pi\right| \text {. }
$$

Therefore $\mathcal{X}_{n_{2}}$ is not unbeatable on $\Pi$.

Case $n=7$. Then $\Pi=(2,2,2,1)$ and $\left|X_{n_{2}} \cap \Pi\right|=15$. There is only one class of transitive (primitive) maximal subgroups of $G=S_{7}$ (not containing $A_{7}$ ). They have order 42 and their intersection with $\Pi$ has size 7 .

Case $n=9$. Then $\Pi=(8,1)$ and $\left|X_{n_{2}} \cap \Pi\right|=7$ !, which is larger than 432 , the maximal size of a primitive subgroup of $S_{9}$ not containing $A_{9}$. Since subgroups in $\mathcal{W}_{3}$ have trivial intersection with $\Pi$, we have that $\mathcal{X}_{n_{2}}$ is unbeatable on $\Pi$.

Case $n=10$. Then $\Pi=(4,4,2)$ and if $M \in \mathcal{W}_{5}$ we have, by Lemma 6 .

$$
|M \cap \Pi|=1.800>1.260=\left|X_{n_{2}} \cap \Pi\right|,
$$

which shows that $\mathcal{X}_{n_{2}}$ is not unbeatable on $\Pi$.

Case $n=11$. We have that $\Pi=(4,4,2,1)$ and $\left|X_{n_{2}} \cap \Pi\right|=56.700$, which is larger than 110, the maximal size of a primitive subgroup of $S_{11}$ not containing $A_{11}$.

Case $n=13$. Then $\Pi=(4,4,4,1)$ and $\left|X_{n_{2}} \cap \Pi\right| \geq 1,24 \cdot 10^{6}$. This number is larger than the maximal size of a primitive subgroup of $S_{13}$ not containing $A_{13}$, which is 156 .

Case $n=14$. Then $\Pi=(8,4,2)$ and $\left|X_{n_{2}} \cap \Pi\right| \geq 1,4 \cdot 10^{7}$. If $M$ is a primitive maximal subgroup of $S_{14}$ not containing $A_{14}$ then $|M \cap \Pi|<|M|<3^{14}<\left|X_{n_{2}} \cap \Pi\right|$. Assume that $M$ is imprimitive. If $M \in \mathcal{W}_{2}$, then

$$
|M \cap \Pi| \leq|M|=2^{7} \cdot 7 !=645.120<\left|X_{n_{2}} \cap \Pi\right| .
$$

If $M \in \mathcal{W}_{7}$ then by Lemma 6 .

$$
|M \cap \Pi|=\frac{|\Pi|}{|G: M|} \cdot 2^{2}=3.175 .200<\left|X_{n_{2}} \cap \Pi\right| .
$$

Case $n=18$. Then $\Pi=(8,8,2)$ and $\left|X_{n_{2}} \cap \Pi\right|=\frac{16 !}{2^{7}} \geq 1,63 \cdot 10^{11}$. If $M$ is a primitive maximal subgroup of $S_{18}$ not containing $A_{18}$, then

$$
|M \cap \Pi|<|M|<3^{18}=387.420 .489<\left|X_{n_{2}} \cap \Pi\right| \text {. }
$$

If $M$ is an imprimitive maximal subgroup of $S_{18}$, not in $\mathcal{W}_{9}$, then

$$
|M \cap \Pi|<|M| \leq(6 !)^{3} \cdot 3 ! \leq 2,24 \cdot 10^{9}<\left|X_{n_{2}} \cap \Pi\right| .
$$

If $M \in \mathcal{W}_{9}$ then by Lemma 6 .

$$
|M \cap \Pi|=\frac{|\Pi|}{|G: M|} 2^{2} \leq 4,12 \cdot 10^{9}<\left|X_{n_{2}} \cap \Pi\right| .
$$

Case $n=20$. Then $\Pi=(8,8,4)$ and $\left|X_{n_{2}} \cap \Pi\right|=\frac{16 !}{2^{7}} \cdot 3 ! \geq 9,8 \cdot 10^{11}$. If $M$ is a primitive maximal subgroup of $S_{20}$ not containing $A_{20}$, then

$$
|M \cap \Pi| \leq 3^{20} \leq 3,49 \cdot 10^{9}<\left|X_{n_{2}} \cap \Pi\right| .
$$

If $M$ is an imprimitive maximal subgroup of $G$, not in $\mathcal{W}_{10}$, then

$$
|M \cap \Pi| \leq|M| \leq\left|S_{5} 2 S_{4}\right|=(5 !)^{4} 4 ! \leq 4,98 \cdot 10^{9}<\left|X_{n_{2}} \cap \Pi\right| .
$$

If $M \in \mathcal{W}_{10}$ then by Lemma 6 ,

$$
|M \cap \Pi|=\frac{|\Pi|}{|G: M|} 2^{2} \leq 2,06 \cdot 10^{11}<\left|X_{n_{2}} \cap \Pi\right| .
$$


Case $n=40$. Then $\Pi=(16,16,8)$ and $\left|X_{n_{2}} \cap \Pi\right|=\frac{32 !}{2^{9}} \cdot 7 ! \geq 2,59 \cdot 10^{36}$. If $M$ is a primitive maximal subgroup of $S_{40}$ not containing $A_{40}$ then $|M \cap \Pi|<|M| \leq$ $2^{40} \approx 1,10 \cdot 10^{12}<\left|X_{n_{2}} \cap \Pi\right|$. If $M$ is an imprimitive maximal subgroup of $G$, not in $\mathcal{W}_{20}$, then

$$
|M \cap \Pi|<|M| \leq\left|S_{10} 2 S_{4}\right|=10 !^{4} \cdot 4 ! \leq 4,17 \cdot 10^{27}<\left|X_{n_{2}} \cap \Pi\right| .
$$

If $M \in \mathcal{W}_{20}$ then by Lemma 6 .

$$
|M \cap \Pi|=\frac{|\Pi|}{|G: M|} 2^{2} \leq 1,16 \cdot 10^{34}<\left|X_{n_{2}} \cap \Pi\right| .
$$

The proof is now complete.

Lemma 10. For $n=10$ we have that $\sigma_{0}\left(S_{10}\right)=46$.

Proof. We already know that $\left\{A_{10}\right\} \cup \mathcal{X}_{2}$ is a primary covering and therefore $\sigma_{0}\left(S_{10}\right) \leq 46$. Assume by contradiction that $\mathcal{C}$ is a primary covering of smaller cardinality. $A_{10}$ belongs to $\mathcal{C}$ because the maximal intersection of a maximal subgroup of $S_{10}$ distinct from $A_{10}$ with the set of the 72.576 elements of cycle structure $(5,5)$ is 576 , realized by the class of subgroups $\mathcal{W}_{5}$, and $72.576 / 576=126>46 \geq \sigma_{0}\left(S_{10}\right)$. To conclude it is enough to show that at least 45 maximal subgroups are needed to cover the elements of cycle type $(4,4,2)$. We were able to do this using the programs [6, 7]. More specifically, this works as follows. Let $\mathscr{M}$ be the set of maximal subgroups of $G=S_{10}$ and let $C$ be the conjugacy class of elements of cycle structure $(4,4,2)$ in $G$. Let $r_{M}$ be a variable for every $M \in \mathscr{M}$ and, for every $x \in C$, define $\mathscr{M}_{x}:=\{M \in \mathscr{M}: x \in M\}$. We found that

$$
\min \left\{\sum_{M \in \mathscr{M}} r_{M} \mid r_{M} \in\{0,1\} \forall M \in \mathscr{M}, \sum_{M \in \mathscr{M}_{x}} r_{M} \geq 1 \forall x \in C\right\}=45 .
$$

For this, [6] was used to compute the sets $\mathscr{M}_{x}$ and [7] was used to solve the optimization problem.

We can now complete this case.

Proposition 4. Assume that $n \neq 10$ and $n \neq 3^{\epsilon} 2^{a}$, for every $\epsilon \in\{0,1\}$ and every $a \geq 0$. Then $\sigma_{0}\left(S_{n}\right)=1+\left(\begin{array}{c}n \\ n_{2}\end{array}\right)$.

Proof. The case $n=5$ has been done in Lemma 8

By Propositions 2 and 3 we have that

$$
\sigma_{0}\left(S_{n}\right) \geq \sigma(\Pi)=\left|\mathcal{X}_{n_{2}}\right|=\left(\begin{array}{c}
n \\
n_{2}
\end{array}\right)
$$

Moreover, if it were $\sigma_{0}\left(S_{n}\right)=\left(\begin{array}{c}n \\ n_{2}\end{array}\right)$ then, by the strongly definitely unbeatable property, $\mathcal{X}_{n_{2}}$ would be a primary covering for $S_{n}$, which is impossible by [3, Theorem 1$]$, or simply because this collection does not cover the primary elements acting fixedpoint-freely. Therefore, $\sigma_{0}\left(S_{n}\right)>\left(\begin{array}{c}n \\ n_{2}\end{array}\right)$ and then Lemma 7 completes the proof. Note that in this situation a minimal primary covering is given by $\left\{A_{n}\right\} \cup \mathcal{X}_{n_{2}}$.

Finally, assume now that $n=3 \cdot 2^{a}$ for some $a \geq 0$. The case $n=3$ is trivial, thus assume that $a \geq 1$. We first deal with the case $n=6$. 
Lemma 11. The primary covering number for $S_{6}$ is 7 . A minimal primary covering is (a conjugate to) the following

$$
\mathcal{C}=\left\{A_{6}, X_{1}, X_{1}^{(12)}, X_{1}^{(13)}, P_{1}, P_{1}^{(34)}, P_{1}^{(35)}\right\},
$$

where $X_{1}=\operatorname{Stab}_{S_{6}}(\{1\}) \in \mathcal{X}_{1}$ and $P_{1}=\langle(3465),(123)(456)\rangle$ belongs to the family $\mathcal{P}$ of primitive maximal subgroups isomorphic to $S_{5}$.

Proof. A direct check with GAP shows that $\mathcal{C}$ is a covering for the set of primary elements of $S_{6}$.

Assume by contradiction that $\mathcal{D}$ is a primary covering (consisting of maximal subgroups of $G$ and) containing less than seven elements.

We first show that $A_{6} \in \mathcal{D}$. If this is not the case, then the class $\Pi_{0}$ of 5 -cycles should be covered by at most six maximal subgroups, which are either 1-point stabilizers, that is elements of $\mathcal{X}_{1}$, or primitive maximal subgroups, that is elements of $\mathcal{P}$, and in both cases they are all isomorphic to $S_{5}$. Note that $\left|\Pi_{0}\right|=6 \cdot 24$, and that for every $S_{1} \neq S_{2} \in \mathcal{X}_{1}$ and every $P_{1} \neq P_{2} \in \mathcal{P}$ we have:

- $\left|\Pi_{0} \cap S_{1}\right|=\left|\Pi_{0} \cap P_{1}\right|=24$,

- $\left|\Pi_{0} \cap S_{1} \cap S_{2}\right|=\left|\Pi_{0} \cap P_{1} \cap P_{2}\right|=0$,

- $\left|\Pi_{0} \cap S_{1} \cap P_{1}\right|=4$.

(the second equation is trivial for the 1-point stabilizers, and it holds for the members of $\mathcal{P}$ too, since there is an outer involutory automorphism of $S_{6}$ that interchanges 1-point stabilizers with the members of $\mathcal{P}$ ).

By applying an inclusion/exclusion argument there are only two ways to cover $\Pi_{0}$ with no more than six of these proper subgroups, either using all of the six elements of $\mathcal{X}_{1}$, or all of the elements of $\mathcal{P}$. It follows that $\mathcal{D}$ is either $\mathcal{X}_{1}$ or $\mathcal{P}$. In both cases we have a contradiction, since $\mathcal{X}_{1}$ does not cover the 2-elements of type $\Pi_{1}=(2,2,2)$, while $\mathcal{P}$ does not cover the 2 -cycles. We proved therefore that $A_{6} \in \mathcal{D}$.

We set $\mathcal{D}_{1}=\mathcal{D} \backslash\left\{A_{6}\right\}$. The collection $\mathcal{D}_{1}$ consists of at most five subgroups, which should cover the set of odd 2-elements, that is the set $\Pi_{1} \cup \Pi_{2} \cup \Pi_{3}$, where $\Pi_{1}=(2,2,2), \Pi_{2}=(4,1,1)$ and $\Pi_{3}=(2,1,1,1,1)$. The following table shows the sizes of the intersections of these classes with the maximal subgroups (different form $\left.A_{6}\right)$.

\begin{tabular}{|c|c|c|c|c|c|c|}
\hline$\Pi_{i}$ & $\left|\Pi_{i}\right|$ & $\left|\Pi_{i} \cap \mathcal{X}_{1}\right|$ & $\left|\Pi_{i} \cap \mathcal{X}_{2}\right|$ & $\left|\Pi_{i} \cap \mathcal{W}_{3}\right|$ & $\left|\Pi_{i} \cap \mathcal{W}_{2}\right|$ & $\left|\Pi_{i} \cap \mathcal{P}\right|$ \\
\hline$(2,2,2)$ & 15 & 0 & 3 & 6 & 7 & 10 \\
$(4,1,1)$ & 90 & 30 & 6 & 0 & 6 & 30 \\
$(2,1,1,1,1)$ & 15 & 10 & 7 & 6 & 3 & 0 \\
\hline
\end{tabular}

We claim that in order to cover the class $\Pi_{2}$ of 4 -cycles we need to take either at least three different elements of $\mathcal{X}_{1}$ or at least three different elements of $\mathcal{P}$. This comes from the fact that, for every $S_{1} \neq S_{2} \in \mathcal{X}_{1}$ and every $P_{1} \neq P_{2} \in \mathcal{P}$, the following holds

- $\left|\Pi_{2} \cap S_{1} \cap S_{2}\right|=\left|\Pi_{2} \cap P_{1} \cap P_{2}\right|=6$,

- $\left|\Pi_{2} \cap S_{1} \cap P_{1}\right|=10$,

- $\left|\Pi_{2} \cap S_{1} \cap S_{2} \cap P_{1}\right|=\left|\Pi_{2} \cap S_{1} \cap P_{1} \cap P_{2}\right|=2$,

- $\left|\Pi_{2} \cap S_{1} \cap S_{2} \cap P_{1} \cap P_{2}\right| \leq 2$,

hence

$$
\left|\Pi_{2} \cap\left(S_{1} \cup S_{2} \cup P_{1} \cup P_{2}\right)\right| \leq 76 .
$$


Assume that $\mathcal{D}_{1}$ contains three different elements of $\mathcal{P}$, then, by looking at the last line of the Table, the class $\Pi_{3}$ should be covered using just two different subgroups, say $A, B \in \mathcal{X}_{1} \cup \mathcal{X}_{2} \cup \mathcal{W}_{3}$. this is impossible, since:

- if $A, B \in \mathcal{X}_{1}$, then $\left|\Pi_{3} \cap A \cap B\right|=6$,

- if $A \in \mathcal{X}_{1}$ and $B \in \mathcal{X}_{2}$, then $\left|\Pi_{3} \cap A \cap B\right| \geq 4$,

- if $A \in \mathcal{X}_{1}$ and $B \in \mathcal{W}_{3}$, then $\left|\Pi_{3} \cap A \cap B\right|=4$.

The opposite case when $\mathcal{D}_{1}$ contains three different elements of $\mathcal{X}_{1}$, follows immediately by using the duality of the outer automorphism of order two of $S_{6}$ (or with similar arguments applied to the first line of the table).

Lemma 12. Let $a_{1}, \ldots, a_{r}$, a be integers with $0 \leq a_{1} \leq \ldots \leq a_{r}, r \geq 2$ and $a \geq 1$.

(1) If $\sum_{i=1}^{r} 2^{a_{i}}=2^{a}$ then there exists a subset $J \subseteq\{1, \ldots, r\}$ such that $\sum_{i \in J} 2^{a_{i}}=2^{a-1}$.

(2) If $\sum_{i=1}^{r} 2^{a_{i}}=3 \cdot 2^{a}$ then one of the following occurs.

- $r=2, a_{1}=a, a_{2}=a+1$.

- $r=3, a_{1}=a_{2}=a_{3}=a$.

- There exist two disjoint subsets $J_{1}, J_{2} \subseteq\{1, \ldots, r\}$ such that

$$
\sum_{i \in J_{1}} 2^{a_{i}}=\sum_{i \in J_{2}} 2^{a_{i}}=2^{a-1} .
$$

Proof. Item (1). Assume that what we want to prove is false, and let $r$ be minimal with the property that $\sum_{i=1}^{r} 2^{a_{i}}=2^{a}$ and there is no $J \subseteq\{1, \ldots, r\}$ with $\sum_{i \in J} 2^{a_{i}}=2^{a-1}$. This implies $r \geq 3$. Note that since twice a power of 2 is a power of 2 , we have $a_{1}<\ldots<a_{r} \leq a-2$, hence

$$
2^{a}=\sum_{i=1}^{r} 2^{a_{i}} \leq \sum_{i=0}^{a-2} 2^{i} \leq 2^{a-1}-1
$$

a contradiction.

Item (2). Assume that $\sum_{i=1}^{r} 2^{a_{i}}=3 \cdot 2^{a}$. By item (1), to conclude it is enough to show that there exists $J \subseteq\{1, \ldots, r\}$ such that $\sum_{i \in J} 2^{a_{i}}=2^{a}$, so suppose this is not the case, by contradiction. As for item (1), we may assume that $a_{1}<\ldots<a_{r}$. We know that $a_{r} \leq a+1$ and $a_{r-1} \neq a$, therefore

$$
3 \cdot 2^{a}=\sum_{i=1}^{r} 2^{a_{i}} \leq \sum_{i=1}^{r-1} 2^{a_{i}}+2^{a+1} \leq \sum_{i=0}^{a-1} 2^{i}+2^{a+1} \leq 2^{a}-1+2^{a+1},
$$

a contradiction.

Proposition 5. Let $n=2^{a+1}+2^{a}=3 \cdot 2^{a}$, with $a \geq 2$. Then

$$
c_{1} \leq \sigma_{0}\left(S_{n}\right) \leq c_{2},
$$

where

$$
\begin{aligned}
& c_{1}= \begin{cases}117 & \text { if } a=2, \\
1+\left(\begin{array}{c}
n-1 \\
2^{a}-1
\end{array}\right) & \text { if } a \geq 3,\end{cases} \\
& c_{2}=2+\left(\begin{array}{c}
n-1 \\
2^{a}-1
\end{array}\right)+\sum_{i=2}^{2^{a+1}}\left(\begin{array}{c}
n-i \\
2^{a-1}-1
\end{array}\right)
\end{aligned}
$$


Proof. To prove the upper bound we consider the collection

$$
\mathcal{C}_{2}=\bigcup_{i=0}^{m} \mathcal{M}_{i}
$$

where $m=2^{a+1}+1$ and

$$
\begin{aligned}
\mathcal{M}_{0} & =\left\{A_{n}\right\}, \\
\mathcal{M}_{1} & =\left\{\operatorname{Stab}_{S_{n}}(U)|1 \in U,| U \mid=2^{a}\right\}, \\
\mathcal{M}_{i} & =\left\{\operatorname{Stab}_{S_{n}}(V)|i \in V \subset\{i, \ldots, n\},| V \mid=2^{a-1}\right\}, \text { for } i=2, \ldots, 2^{a+1}, \\
\mathcal{M}_{m} & =\left\{\operatorname{Stab}_{S_{n}}(\{m, \ldots, n\})\right\} .
\end{aligned}
$$

The primary elements of odd order as well as the 2-elements that are even permutations are covered by $A_{n}$. Let $g$ be an odd 2-element. If $g \in\left(2^{a}, 2^{a}, 2^{a}\right)$ then $g$ is covered by a unique subgroup in $\mathcal{M}_{1}$. Otherwise, since $g$ is an odd permutation, by Lemma 12 there are at least two disjoint subsets of cardinality $2^{a-1}$ on which $g$ acts. If one of these two contains the point 1 , then again $g$ is covered by a unique element of $\mathcal{M}_{1}$, otherwise $g$ lies in the stabilizer of a subset of size $2^{a-1}$ and not containing 1 . In this case $g$ is covered by a subgroup that lies in $\bigcup_{i=2}^{m} \mathcal{M}_{i}$. Since $\left|\mathcal{C}_{2}\right|=c_{2}$ the upper bound is proved.

We prove now that $c_{1}$ is a lower bound for $\sigma_{0}\left(S_{n}\right)$.

Assume first that $a=2$, that is $n=12$. Arguing in a similar way as the cases $n=5,6$ or 10, it is straightforward to prove that the alternating group $A_{12}$ belongs to every minimal primary covering. Now, the maximal subgroups that contain most elements of type $\Pi_{1}=(4,4,4)$ are the ones in $\mathcal{W}_{6}$, each of which, by Lemma 6] contains exactly $8\left|\Pi_{1}\right|(6 !)^{2} / 12$ ! such elements. Since $12 ! / 8(6 !)^{2}=115,5$ we conclude that a minimal primary covering has at least 117 elements.

Let $a \geq 3$. We prove that $c_{1}$ is a lower bound for $\sigma_{0}\left(S_{n}\right)$ by showing that the collection $\mathcal{M}_{1}$ is definitely unbeatable on $\Pi_{1}=\left(2^{a}, 2^{a}, 2^{a}\right)$.

Conditions (1) and (2) of Definition 1 follow immediately. Let us prove condition (3). Note that the only maximal subgroups $M$ having nontrivial intersection with $\Pi_{1}$ are either stabilizers of a set of cardinality $2^{a}$, or imprimitive, or proper primitive maximal subgroups, that is elements of $\mathcal{W} \cup \mathcal{P}$. For such $M$ we define $c(M):=$ $\left|M \cap \Pi_{1}\right| /\left|M_{1} \cap \Pi_{1}\right|$ for $M_{1} \in \mathcal{M}_{1}$, and we will prove that $c(M) \leq 1$.

In the first case we have that

$$
\left|M \cap \Pi_{1}\right|=\left|M_{1} \cap \Pi_{1}\right|=\frac{\left(2^{a} !\right)\left(2^{a+1} !\right)}{2^{3 a+1}},
$$

for every $M_{1} \in \mathcal{M}_{1}$ and every $M=\operatorname{Stab}_{S_{n}}(U), 1 \notin U,|U|=2^{a}$. Therefore $c(M)=1$.

Assume $M \in \mathcal{W}_{n / 2}$. Then by Lemma 6 .

$$
\left|M \cap \Pi_{1}\right|=\frac{4\left|\Pi_{1}\right|\left|W_{n / 2}\right|}{n !}=\frac{4}{3 \cdot 2^{3 a}}((n / 2) !)^{2},
$$


and therefore

$$
\begin{aligned}
c(M) & =\frac{8}{3} \cdot \frac{((n / 2) !)^{2}}{\left(2^{a+1}\right)\left(2^{a} !\right)}=\frac{8}{3} \cdot \frac{\left(\left(2^{a}+2^{a-1}\right) !\right)^{2}}{\left(2^{a+1}\right) !\left(2^{a}\right) !} \\
& =\frac{8}{3} \cdot \frac{(3 t !)^{2}}{(4 t) !(2 t) !}=\frac{8}{3} \cdot \frac{\left(\begin{array}{c}
6 t \\
2 t
\end{array}\right)}{\left(\begin{array}{l}
6 t \\
3 t
\end{array}\right)}
\end{aligned}
$$

where $t=2^{a-1}$. Therefore $c(M)<1$ for every $t \geq 4$, that is for every $a \geq 3$.

Assume $M \in \mathcal{W}_{d}$, with $d \neq n / 2$. Then $\left|M \cap \Pi_{1}\right|<|M| \leq\left|W_{n / 3}\right|$ by Lemma [5] therefore

$$
\begin{aligned}
c(M) & <\frac{\left|W_{n / 3}\right|}{\left|M_{1} \cap \Pi_{1}\right|}=\frac{(n / 3 !)^{3} \cdot 6 \cdot 2^{3 a+1}}{\left(2^{a} !\right)\left(2^{a+1} !\right)} \\
& <\frac{2^{3 a+4}}{\left(\begin{array}{c}
2^{a+1} \\
2^{a}
\end{array}\right)} \leq \frac{2^{3 a+4}\left(2^{a+1}+1\right)}{2^{2^{a+1}}}<\frac{2^{4 a+6}}{2^{2^{a+1}}} \leq 1,
\end{aligned}
$$

for every $a \geq 4$. The case $a=3$ can be checked directly.

Finally assume that $M \in \mathcal{P}$. If $a \geq 3$, then $|M| \leq 2^{n}$ by [13], hence

$$
c(M)<\frac{|M|}{\left|M_{1} \cap \Pi_{1}\right|}<\frac{2^{n+3 a+1}}{\left(2^{a+1} !\right)\left(2^{a} !\right)}<1 .
$$

The proof is completed.

Acknowledgments. The authors are grateful to the referee for completing the calculations of $\sigma_{0}\left(S_{10}\right)$ using [6, 7].

\section{REFERENCES}

[1] Cohn, J. H. E., On n-sum groups, Math. Scand. Volume 75, Number 1, Year 1944, Pages $44-58$.

[2] Dixon, John D., Mortimer, Brian, Permutation groups. Graduate Texts in Mathematics, 163. Springer-Verlag, New York, 1996.

[3] Fein, B. and Kantor, W. M. and Schacher, M., Relative Brauer groups. II, J. Reine Angew. Math., Volume 328, Year 1981, Pages 39-57.

[4] Fumagalli, F., On the indices of maximal subgroups and the normal primary coverings of finite groups. J. of Group Theory, Volume 22, Number 6, Year 2019, Pages 1015-1034.

[5] Gaschütz, W., Die Eulersche Funktion endlicher auflösbarer Gruppen. Illinois J. Math., Volume 3, Year 1959, Pages 469-476.

[6] The GAP Group. GAP - Groups, Algorithms, and Programming, Version 4.7.5, 2014.

[7] Gurobi Optimizer Reference Manual, Gurobi Optimization, Inc., 2014, (http://www.gurobi.com).

[8] Huppert, B., Endliche Gruppen. I, Die Grundlehren der Mathematischen Wissenschaften, Band 134, Year 1967, Pages xii+793.

[9] Isaacs, I. Martin, Finite Group Theory. Graduate Studies in Mathematics, 92. American Mathematical Society, Providence, RI, 2008. xii+350 pp.

[10] Jones, G. A., Cyclic regular subgroups of primitive permutation groups, J. Group Theory, Volume 5, Year 2002, Number 4, Pages 403-407.

[11] Kappe, Luise-Charlotte and Nikolova-Popova, Daniela and Swartz, Eric, On the covering number of small symmetric groups and some sporadic groups, Groups Complex. Cryptol., Volume 8, Year 2016, Number 2, Pages 135-154.

[12] Maróti, A., Covering the symmetric groups with proper subgroups, J. Combin. Theory Ser. A, Volume 110, Year 2005, Number 1, Pages 97-111.

[13] Maróti, A., On the orders of primitive groups, J. Algebra, Volume 258, Year 2002, Number 2, Pages 631-640.

[14] Oppenheim, Ryan and Swartz, Eric, On the covering number of $S_{14}$, Involve, Volume 12, Year 2019, Number 1, Pages 89-96, ISSN 1944-4176. 
[15] Robinson, Derek J.S., A course in the theory of groups. Second edition. Graduate Texts in Mathematics, 80. Springer-Verlag, New York, 1996.

[16] Swartz, E., On the covering number of symmetric groups having degree divisible by six, Discrete Mathematics, Volume 339, Year 2016, Number 11, Pages 2593-2604.

[17] Tomkinson, M. J., Groups as the union of proper subgroups, Math. Scand., Volume 81, Year 1997, Number 2, Pages 191-198. 